\title{
Light Sensor Platform Based on the Integration of Bacteriorhodopsin with a Single Electron Transistor
}

\author{
Karl A. Walczak, ${ }^{1}$ Paul L. Bergstrom, ${ }^{2}$ and Craig R. Friedrich ${ }^{1}$ \\ ${ }^{1}$ Department of Mechanical Engineering-Engineering Mechanics, Multi-Scale Technologies Institute, \\ Michigan Technological University, Houghton, MI 49931-1295, USA \\ ${ }^{2}$ Department of Electrical and Computer Engineering, Multi-Scale Technologies Institute, \\ Michigan Technological University, Houghton, MI 49931-1295, USA \\ Correspondence should be addressed to Karl A. Walczak, kawalcza@mtu.edu
}

Received 11 January 2011; Accepted 6 April 2011

Academic Editor: Abdelkarim Mercha

Copyright (๑) 2011 Karl A. Walczak et al. This is an open access article distributed under the Creative Commons Attribution License, which permits unrestricted use, distribution, and reproduction in any medium, provided the original work is properly cited.

\begin{abstract}
This paper reports on the integration of an optical protein with single electron transistors to form a nano-bio-hybrid device for sensing. Bacteriorhodopsin (bR) is an optoelectric protein that translocates a proton across a distance of several nanometers in response to an absorbed photon of incident light. This charge gradient results in a measurable voltage in the dried state. Single electron transistors (SETs) have active regions consisting of one or more quantum islands with a size typically 10 nanometers or less. Integrating bacteriorhodopsin with the gate of a SET provides a device capable of a modulated electrical output in response to optical modulation at the device gate. Modulation of the optoelectric activity of the bR by chemical binding with a targeted environmental antigen can form a direct chemical-to-electrical sensor reducing the size and complexity of fluorescencebased systems. The work resulted in electrical resistance and capacitance characterization of purple membrane containing bR under variable illumination to ensure minimal impact on SET operation. Purple membrane containing bacteriorhodopsin was electrodeposited on the SET gates, and current throughput was well correlated with variable and cyclic illumination. It was confirmed that bR optoelectric activity is capable of driving SETs.
\end{abstract}

\section{Introduction}

Size, cost, and pervasiveness of sensing systems are key drivers in their development. One approach that may reduce the size and cost is to integrate an optoelectric transduction material directly with nanoelectronic devices, such as single electron transistors. If binding between an antibodyfunctionalized transduction material and an antigen in the environment alters the electrical activity of the transduction material on the gates of multiple SETs, then this compact sensor design could save size and cost. This is especially true if many transistors form an array whereby different functional chemistries will bind with different antigens giving many sensing sites on a single chip. This approach differs from functionalized nanowire chips where supplemental circuitry is required to determine changes in nanowire resistance or impedance upon antigen binding. It has been shown that the electrical output of the optical protein bacteriorhodopsin (bR) can be modulated by attaching functionalized semiconductor quantum dots whereby, upon conjugate binding, the bR photovoltage is changed [1]. The same work functionalized bR with quantum dots, maltosebinding protein, and the fluorescence quencher molecule $\beta$-CD-QS9Y. Upon binding with maltose present in the ambient, the fluorescence quencher is preferentially released from the assembly resulting in increased bR photovoltage.

Bacteriorhodopsin is the optoelectric transmembrane protein found in the outer cell membrane of the bacterium Halobacterium salinarum. The bR protein together with a lipid bilayer is collectively referred to as purple membrane (PM). When illuminated with light, bR generates a charge gradient and electrical potential by shifting protons from the cytoplasmic side to the extracellular side of the membrane. This extensively studied protein $[2,3]$ is extraordinarily 
robust and can function in extreme environmental conditions [4] and in aqueous and dried states. Bacteriorhodopsin is a soluble protein that can be chemically linked $[5,6]$ or genetically engineered [7].

The ability to modulate the activity of CMOS devices by $\mathrm{PM}$ and fabrication process development compatible with standard lift-off lithography has been shown [8]. This work showed the ability of bR to modulate CMOS devices in response to changes in illumination too small to be perceptible and measurable on conventional instruments. Purple membrane containing bacteriorhodopsin has been integrated with semiconductor-based microdevices [9-11], gallium arsenide field-effect transistors, [12], indium gallium arsenide FET detectors [13], and nanotubes [14], for example. To further reduce the size and power requirements of sensing arrays based on gate modulation, the integration of PM with SETs to form a nano-bio-hybrid system was investigated.

\section{Experimental Section}

2.1. Purple Membrane Preparation. Bacteriorhodopsin is a transmembrane protein, was produced from the SJ9-P strain of Halobacterium salinarum as described by [15] with further centrifuge purification processes, and results in small pieces of the purple membrane containing bR. PM is a two dimensional matrix of bRs and phospholipids so the reported electrical characteristics and SET performance are of this composite material, whereas optoelectric functionality and activation of the SET is a result of the bR itself.

2.2. SET-PM Electrical Equivalent. The SETs used in this work had the side-gate electrically connected to a PMcoated terminal as shown schematically in Figure 1. A SET exploits the quantum mechanical properties of electrons to control the current flowing from the source to the drain terminal. The function of room temperature SETs is based on the tunneling of electrons across insulating energy barriers and tungsten quantum islands less than $10 \mathrm{~nm}$ in diameter situated between the source and drain [16]. Changing the gate potential of the SET changes the tunneling probability and rate of electron flow from the source to the drain terminal via the islands. Ideal SET operation depends on the modulation of charge on the gate terminal with no flow of charge from the gate to the drain through the quantum islands. The minimum current flow, or off state of the SET is located in the Coulomb blockade region of the current versus voltage $(I V)$ curve. Coulomb blockade is a phenomenon which occurs when there is no tunneling of electrons between the source, quantum islands, and drain. Coulomb blockade can be removed by changing the source bias voltage and/or by adding a gate voltage to align the energy levels on the quantum islands with those of the source and drain [17]. Ideal SETs have only one accurately placed sub-10 nm quantum island/dot. However, in this work there are multiple tungsten quantum islands in the SET active region which can tend to blur the blockade effect resulting in source-drain current leakage in the nanoamp range.
One method of integrating PM with a SET is to deposit the PM onto the SET side-gate, or onto a larger secondary pad that is electrically connected to the gate. This second approach permits a larger area for PM and a coupled antibody or functionalized semiconductor quantum dots integrated with the bR for increased antigen binding probability. These functionalized semiconductor quantum dots should not be confused with the quantum islands of the SET. Antigen binding to the UV-illuminated functionalized semiconductor quantum dots linked to the bR within the PM should have their photonic output quenched by the antigen, or the antigen may preferentially displace a dark quencher molecule attached to the quantum dots thereby permitting the quantum dots to radiate photons, as previously demonstrated [18].

Incorporating PM with a SET first had to be analyzed to demonstrate modulation by $\mathrm{bR}$ and determine if the PM would significantly alter the functionality of the SET, primarily through a change in gate capacitance and more importantly the device charging energy given by

$$
\left[E_{c}=\frac{\mathrm{e} 2}{2 C^{*}}\right]
$$

where $E_{c}$ is the charging energy, e is the electron charge, and $C^{*}$ is the combined equivalent capacitance of the quantum islands and device terminals. The devices used in this study were assumed to be similar to those of Karre since they were fabricated using the same equipment and techniques. Those devices had a charging energy measured to be approximately $160 \mathrm{meV}$, about five times that of the room temperature thermal noise. As can be seen from (1) the charging energy will decrease in direct proportion to an increase of the device capacitance caused by integrating PM on the gate terminal or by adding a larger secondary sensing terminal with PM. Low charging energy can cause unwanted spontaneous activation of the SET. The overall capacitance of the type of SET used in these experiments was reported to be approximately $0.5 \mathrm{aF}$ [19]. Therefore, a minimal increase in device capacitance will decrease the device charging energy to near ambient thermal noise possibly resulting in spontaneous current modulation.

Purple membrane with bR was modeled as a charge and voltage source with a resistance and capacitance in parallel [20]. A schematic equivalent of the combined PM-SET bionano-hybrid device is shown in Figure 2 and is based on the orthodox model [21] of a SET and the model of PM. Each of these components has individual theoretical and/or empirical models but here they are combined as a single nano-bio-hybrid device.

In Figure 2(a), $R$ represents the SET gap tunneling resistances of the alumina substrate onto which tungsten source and drain electrodes were patterned by focused ion beam machining and $R_{g}$ is the gate gap resistance. $C$ represents the source-drain gap capacitances and $C_{g}$ is the gate gap capacitance. This type of SET uses multiple quantum islands; therefore, the effective tunneling resistance and capacitance for the multiple dots is made a part of the source-drain tunneling resistance and capacitance. In Figure 2(b), $R_{\text {eqbR }}$ and $C_{\text {eqbR }}$ represent the equivalent resistance and capacitance of the PM layer on the gate or 


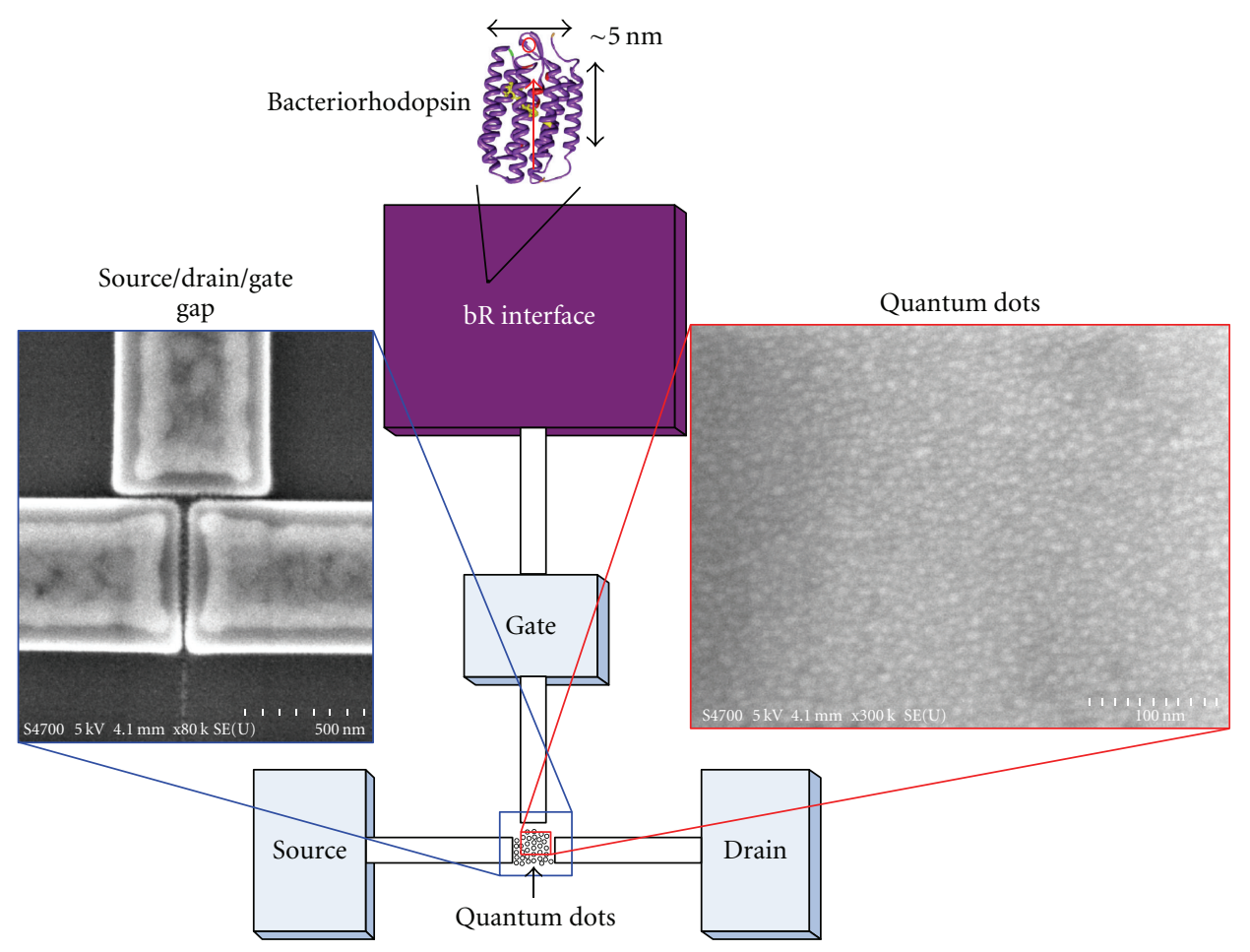

FIGURE 1: Side-gate single electron transistor coupled with purple membrane.

Model SET Model bR Model bR/SET
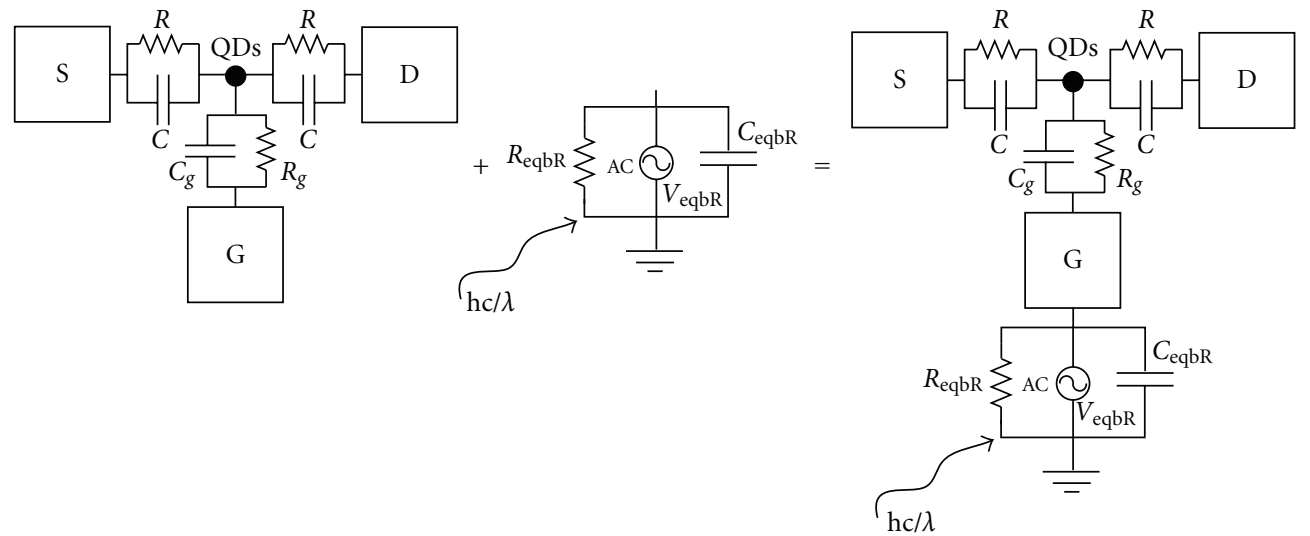

(a)

(b)

(c)

FIGURE 2: Schematic of (a) single electron transistor showing equivalent resistance and capacitance of quantum islands, substrate, and gate; (b) bacteriorhodopsin and lipid bilayer forming the purple membrane; (c) the combined bR/SET.

the larger pad attached to the gate, and $V_{\text {eqbR }}$ is the potential generated by the bR upon illumination. The right portion of Figure 2(c) shows the combined hybrid equivalent. The prior studies by Karre determined the relevant SET parameters of Figure 2(a), whereas the present work resulted in the PM electrical characteristics of Figure 2(b) required to complete this model.

2.3. PM Electrical Properties Measurements. The effective capacitance $C_{\text {eqbR }}$ and resistance $R_{\text {eqbR }}$ were measured to determine the PM electrical characteristics important for SET integration. There has been some work conducted on measuring the electronic properties of PM film but most have been performed in the aqueous state [22-24]. In the present work, tests were performed on dried films of PM to determine if there is a relationship between membrane patch physical orientation, light-on/off amplitude and frequency, and test duration on the effective resistance and capacitance of the PM. Because bR occurs in patches of the original bacterial membrane and PM has a charge differential across 


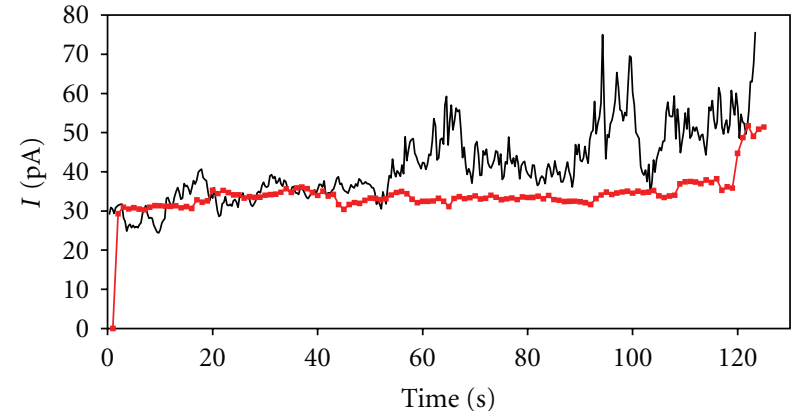

- Without light

$\rightarrow$ With light flashing

FIGURE 3: Baseline measurements source-drain current of SET without bR deposited on the gate at $0.5 \mathrm{~V} \mathrm{~S}$-D bias when illuminated with LED peak intensity at $595 \mathrm{~nm}$ and at various on-off periods ranging from 5 to 60 seconds.

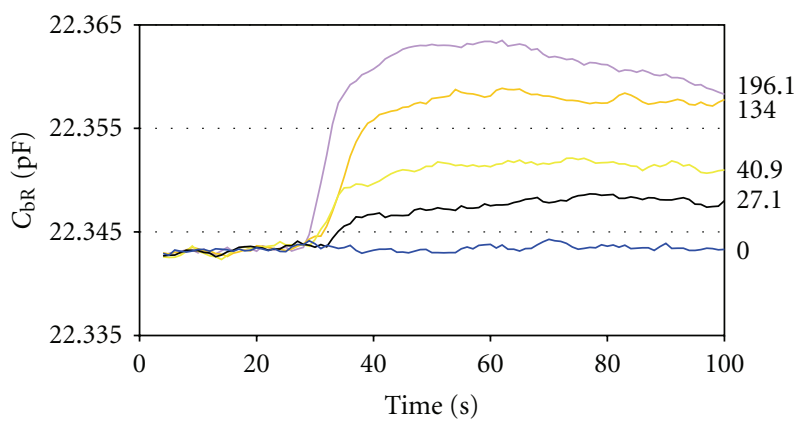

FIgure 4: Capacitance of dried bR $(\mathrm{pF})$ versus time for five LED intensities $\left(\mathrm{W} / \mathrm{m}^{2}\right)$ measured at $570 \mathrm{~nm}$ for $22 \mu \mathrm{m}$ thick unoriented sample. LED peak emission was at $595 \mathrm{~nm}$.

its $6 \mathrm{~nm}$ thickness above $\sim \mathrm{pH} 5$, the patches can be oriented so that the vectorial charge gradients are additive.

Indium tin oxide- (ITO-) coated glass substrates covered with PM were created by depositing $20 \mu \mathrm{L}$ of a $6.9 \mathrm{mg} / \mathrm{mL}$ solution of PM in DI water over an area of $3 \mathrm{~mm} \times 3 \mathrm{~mm}$ using the method described in [8]. One set of samples had electrodeposited and oriented PM using electrophoretic deposition at 4 VDC for 40 seconds across a $1 \mathrm{~mm}$ gap thickness between the top electrode and the ITO glass substrate. Unoriented PM samples were prepared by pipetting $20 \mu \mathrm{L}$ of the same PM solution onto ITO glass without electrophoretic deposition. All samples were air dried for two days at $21^{\circ} \mathrm{C}$. The bR photovoltage was measured using a $T \Omega$ input impedance operational amplifier. The samples were activated using amber LEDs with a peak emission at $595 \mathrm{~nm}$. The thickness of each sample was measured using a VEECO Dektak 6M Stylus Profiler. Optical absorbance measurements of the dried samples were made using an Ocean Optics USB4000 spectrometer.

SET electrical measurements were made with an HP 4284A LCR meter controlled through an interface module in a Keithley 4200 semiconductor parametric analyzer. RF noise was reduced by placing the test fixture in a grounded

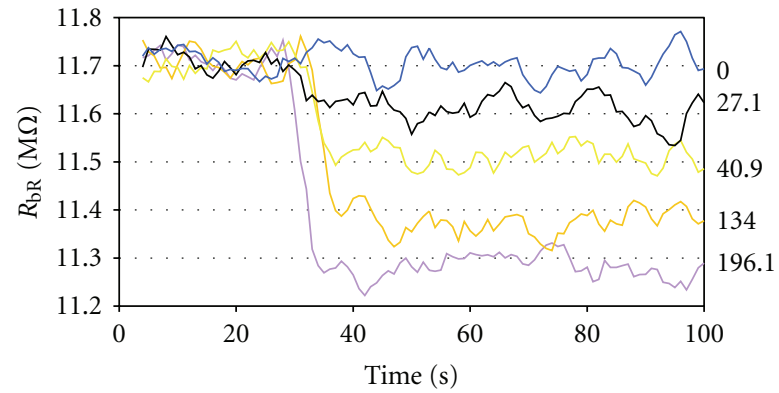

FIgURE 5: Resistance of dried bR $(\mathrm{M} \Omega)$ versus time for five LED intensities $\left(\mathrm{W} / \mathrm{m}^{2}\right)$ measured at $570 \mathrm{~nm}$ for $10 \mu \mathrm{m}$ thick oriented sample. Peak Led emission was at $595 \mathrm{~nm}$.

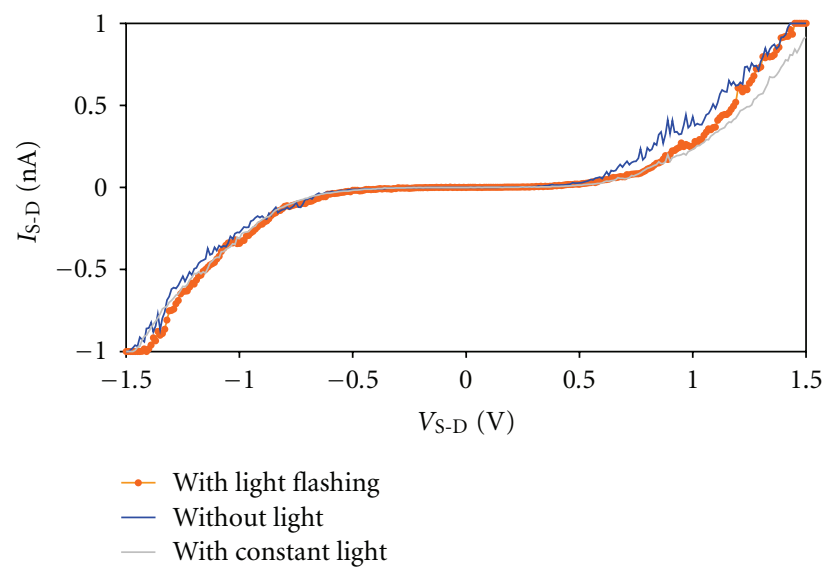

FIGURE 6: Source-drain current versus voltage measurements of a single electron transistor without PM immobilized on the side-gate, with and without constant illumination. Illumination was LED with peak emission at $595 \mathrm{~nm}$.

enclosure. To minimize parasitic capacitance errors in the measurements, open and short circuit correction measurements were performed prior to testing. For each recorded measurement, five consecutive measurements were taken. The LED light intensity was adjusted for five discrete values of $0,27.1,40.9,134.0$, and $196.1 \mathrm{~W} / \mathrm{m}^{2}$ at $570 \mathrm{~nm}$. The LED intensity for the tests was referenced at $570 \mathrm{~nm}$ since this is the peak absorbance of bR.

2.4. SET Fabrication and PM Integration. SETs were fabricated using focused ion beam-induced deposition of tungsten quantum islands on an alumina substrate, followed by sputter deposition and patterning of tungsten source, drain, and gate electrodes. The PM was electrodeposited onto a $3 \mathrm{~mm} \times 3 \mathrm{~mm}$ pad electrically connected to the SET gate (Figure 1) using the same procedure described above for electrical characterization measurements. SET output measurements were made with a Keithley 4200 semiconductor parameter analyzer with a preamp connected to the collector. The SETs were first characterized without PM deposited on the gate to determine if the light would alter the SET functionality due to the generation of photoelectrons. As shown in Figure 3, there is no distinct photoelectrical 


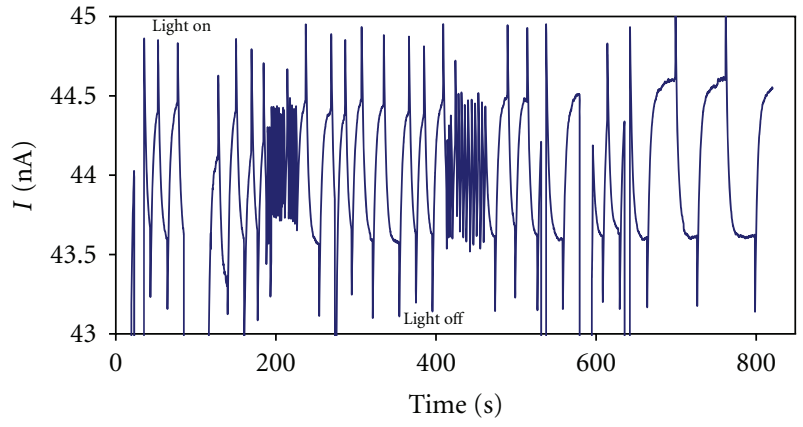

FIGURE 7: Source-drain current of br-SET at $0.5 \mathrm{~V}$ S-D bias when illuminated with LED peak intensity at $595 \mathrm{~nm}$ and at various onoff periods ranging from 5 to 60 seconds. The current bias at approximately $44.1 \mathrm{nA}$ is apparently due to larger quantum islands allowing a constant leakage current across the SET.

response without bR present on the exposed gate of the SET. The PM-SETs were illuminated with amber LEDs over a range of illumination intensity and frequencies that might be characteristic in a sensing system.

\section{Results and Discussion}

The results were obtained to determine the influence of the addition of PM on or near the SET side gate to complete the model prediction on device charging energy, and then the behavior of the hybrid SET was measured after final integration of the PM.

3.1. PM Electrical Properties Relevant to SET Integration. The PM sample capacitance and resistance versus time and light intensity are shown in Figures 4 and 5. The oriented and unoriented samples show similar trends in capacitance and resistance versus time and light intensity. Figure 4 indicates the effective capacitance increases upon light activation and with increasing light intensity. This response for unoriented PM with a thickness of $22 \mu \mathrm{m}$ was nearly identical to oriented PM with a thickness of $10 \mu \mathrm{m}$. As shown in Figure 5, the resistance across the bR decreased with light activation and increasing light intensity. Both results are not surprising and somewhat intuitive. Capacitance is the ability of a material to maintain charge separation and that is what bR does via proton translocation across the membrane. Upon continual illumination, bR is capable of maintaining a $\mathrm{pH}$ change of up to 4 across its thickness, a proton concentration gradient of 10,000 . Additionally, as bR translocates charge it would be expected to see a decrease in effective resistance due to a current flow across the PM deposition thickness, and under constant illumination a steady state current is indicated by a lower resistance.

The measurements showed that there was a change in capacitance upon illumination for both oriented and unoriented samples. These changes appear relatively insignificant considering they were on the order of 5 to $20 \mathrm{fF}$, but when integrated with a SET with a total capacitance approximately $0.5 \mathrm{aF}$, this change in capacitance could be significant and might alter the SET charging energy and functionality. From the measurements of the dried films, it was determined that the dominant electrical characteristics of the PM were the capacitance and resistance for light activated and not activated, but much less so for oriented and unoriented samples. Based on the bR relative permittivity of 2.2 estimated from associated impedance measurements, the PM deposition area and thicknesses, the equivalent capacitance $C_{\text {eqbr }}$ was estimated to be $22 \mathrm{pF}$, several orders of magnitude larger than the SET capacitance. When the PM is placed on the SET gate, the capacitance is in series with the side-gate capacitance. As shown in (2), the capacitance of the PM has no significant effect on the overall device capacitance, which suggests that integrating PM on the gate or a larger sensing pad should have no adverse charging energy effect on the functionality of the SET

$$
\left[\frac{1}{C^{*}}=\frac{1}{C_{g}}+\frac{1}{C_{\mathrm{br}}}=\frac{1}{0.5 \mathrm{aF}}+\frac{1}{22 \mathrm{pF}} \stackrel{C^{*}}{\longrightarrow} 0.5 \mathrm{aF}\right] .
$$

3.2. SET-bR Integration Results. As shown in Figure 6, there appeared to be no significant difference in the SET functionality with and without light. The fluctuation is on the order of $0.1 \mathrm{nA}$ of source-drain current; however, the trend of the data is indicative that the SET may not be completely immune to photonic stimulation. Therefore, any significant source-drain current modulation of the bRSETs when activated by light should be attributable to the influence of the bR with current levels significantly higher than $0.1 \mathrm{nA}$.

Tests were conducted with PM immobilized on the gate interface of SETs activated with LED illumination over a wide range of frequencies that might be characteristic of a sensing system, as shown in Figure 7. The figure shows that the light-activated PM immobilized on the gate interface modulated the SET with good fidelity. The source-drain current response to illumination periods from 5 to $60 \mathrm{sec}-$ onds showed a peak-peak variation of approximately $2 \mathrm{nA}$, providing a signal-noise ratio of approximately 20 when compared with $0.1 \mathrm{nA}$ of source-drain current without bR (Figure 6). The results showed consistent peak-peak current modulation at all intermediate periods and confirmed the current modulation was in response to the intermittent illumination for a range of frequencies that would be characteristic of the sampling frequency in a sensing system. These tests verified that variations of the bR photovoltage were influencing the SET function.

3.3. Discussion. Upon observation, there is an inconsistency between the SET current behavior of the device in Figure 6 and that of Figure 7. The SET device of Figure 6 shows no noticeable current between $\pm 0.5 \mathrm{~V}$ of source-drain voltage with $\mathrm{PM}$ on the gate with different illumination. Beyond $\pm 0.5 \mathrm{~V}_{\mathrm{SD}}$, there are fractional $\mathrm{nA}$ source-drain current changes in response to illumination. This device was fabricated early in the research project and is very similar to those previously reported $[25,26]$. The device used in the results shown in Figure 7 was fabricated much later and is similar to those reported [27]. The reason for the differences 
between the earlier and later devices is that the FIB system source aperture had unknowingly enlarged with extensive use and the tungsten quantum island deposition ion current had increased for the same FIB current setting. In the work of Karre, the FIB had been carefully characterized to get wellbehaved SETs and the device shown in Figure 6 is indicative of this. However, with FIB aperture aging and less control on the size quantum island deposition, more device leakage was apparently occurring. This was also observed and reported by Acharya, using similar devices. It is conjectured that the increased leakage current is due to unintended tunneling to and from nonparticipating quantum islands outside the central functional region of the devices. This is considered the reason the latter device response shown in Figure 7 had a current offset of approximately $44.1 \mathrm{nA}$, admittedly high compared to the device of Figure 6. However, even with this offset variation the drain current is faithfully repeated with the variable frequency illumination fluctuations of the PM-gate structure. All devices were tested to ensure the response was not due to illumination alone without PM attached. This device was no different. This result also reinforces the challenges of fabricating large numbers of repeatable nanoelectronic devices by FIB deposition of quantum islands.

\section{Conclusions}

This research focused on the development of technology that could be used to create a platform for a chemical/biological sensor by integrating bacteriorhodopsin with SETs using electrophoretic deposition on a side-gate conductive pad, but Langmuir-Blodgett and ionic self-assembly are also other potential methods for deposition. PM substrates were used to determine the effective capacitance and resistance of PM under lighting conditions likely to be encountered in a sensor application, and it was found that using PM did not affect the fundamental operation of the SETs. The SETs were fabricated by a combination of sputtering, photolithography, and focused ion beam machining. The gate pads served as the interface for the PM with the SETs. Characterization demonstrated that the bR modulated the operational characteristics of the SETs when the bR was activated with light within its absorbance spectrum with a signal to noise ratio as high as 20 . There was no effort to optimize the SETs or the fabrication process specifically for use with PM. With this initial research into the integration of PM with nanoelectronic devices and model development, it is proposed that this research should be further developed and eventually lead to the development of a multiconfigurable nanoscale sensor array for applications which includes labon-chips or artificial eyes.

\section{Acknowledgments}

The research reported in this document was performed in connection with contract DAAD17-03-C-0115 with the U.S. Army Research Laboratory. The views and conclusions contained in this document are those of the authors and should not be interpreted as presenting the official policies or position, either expressed or implied, of the U.S. Army Research Laboratory or the U.S. Government unless so designated by other authorized documents. Citation of manufacturer's or trade names does not constitute an official endorsement or approval of the use thereof. The U.S. Government is authorized to reproduce and distribute reprints for Government purposes notwithstanding any copyright notation hereon.

\section{References}

[1] M. Griep, E. Winder, D. Lueking, C. Friedrich, G. Mallick, and S. Kama, "Optical protein modulation via quantum dot coupling and use of a hybrid sensor protein," Journal of Nanoscience and Nanotechnology, vol. 10, no. 9, pp. 6029-6035, 2010.

[2] W. Stoeckenius, "From membrane structure to bacteriorhodopsin," Journal of Membrane Biology, vol. 139, no. 3, pp. 139-148, 1994.

[3] N. Hampp, "Bacteriorhodopsin as a photochromic retinal protein for optical memories," Chemical Reviews, vol. 100, no. 5, pp. 1755-1776, 2000.

[4] N. Vsevolodov, Biomolecular Electronics: An Introduction via Photosensitive Proteins, Birkhauser, Boston, Mass, USA, 1998.

[5] M. Schranz, F. Noll, and N. Hampp, "Oriented purple membrane monolayers covalently attached to gold by multiple thiole linkages analyzed by single molecule force spectroscopy," Langmuir, vol. 23, no. 22, pp. 11134-11138, 2007.

[6] T. Pouyani, J. W. Kuo, G. S. Harbison, and G. D. Prestwich, "Solid-state NMR of N-acylureas derived from the reaction of hyaluronic acid with isotopically-labeled carbodiimides," Journal of the American Chemical Society, vol. 114, no. 15, pp. 5972-5976, 1992.

[7] G.-Q. Chen and J. E. Gouaux, "Overexpression of bacterioopsin in Escherichia coli as a water-soluble fusion to maltose binding protein: efficient regeneration of the fusion protein and selective cleavage with trypsin," Protein Science, vol. 5, no. 3, pp. 456-467, 1996.

[8] C. M. Anton, "Photolithography based patterning of bacteriorhodopsin films," in Mechanical Engineering and Engineering Mechanics, p. 186, Michigan Technological University, Houghton, Mich, USA, 2008.

[9] P. Bhattacharya, J. Xu, G. Váró, D. L. Marcy, and R. R. Birge, "Monolithically integrated bacteriorhodopsin-GaAs field-effect transistor photoreceiver," Optics Letters, vol. 27, no. 10, pp. 839-841, 2002.

[10] J. Xu, P. Bhattacharya, D. L. Marcy, J. A. Stuart, and R. R. Birge, "Photoconduction in bacteriorhodopsin/GaAs heterostructures," Electronics Letters, vol. 37, no. 10, pp. 648649, 2001.

[11] J. Shin, P. Bhattacharya, H. C. Yuan, Z. Ma, and G. Váró, "Lowpower bacteriorhodopsin-silicon n-channel metal-oxide fieldeffect transistor photoreceiver," Optics Letters, vol. 32, no. 5, pp. 500-502, 2007.

[12] J. Xu, P. Bhattacharya, and G. Váró, "Monolithically integrated bacteriorhodopsin/semiconductor opto-electronic integrated circuit for a bio-photoreceiver," Biosensors and Bioelectronics, vol. 19, no. 8, pp. 885-892, 2004.

[13] Q. Ren, Y.-P. Zhao, L. Han, and H. B. Zhao, "A nanomechanical device based on light-driven proton pumps," Nanotechnology, vol. 17, no. 6, pp. 1778-1785, 2006. 
[14] G. Gruner, "Carbon nanotube transistors for biosensing applications," Analytical and Bioanalytical Chemistry, vol. 384, no. 2, pp. 322-335, 2006.

[15] D. Oesterhelt and W. Stoeckenius, "Isolation of the cell membrane of halobacterium halobium and its fractionation in to red and purple membrane," in Methods in Enzymology, S. P. Colowick and N. O. Kaplan, Eds., vol. 31, Academic Press, New York, NY, USA, 1974.

[16] P. S. K. Karre, P. L. Bergstrom, G. Mallick, and S. P. Karna, "Room temperature operational single electron transistor fabricated by focused ion beam deposition," Journal of Applied Physics, vol. 102, no. 2, pp. 1-4, 2007.

[17] D. Berman, The aluminum single-electron trnasistor for ultrasensitive electrometry of semiconductor quantum-confined systems, Ph.D. thesis, Department of Electrical Enegineering and Computer Science, MIT, Cambridge, Mass, USA, 1998.

[18] M. H. Griep, "Quantum dot/optical protein bio-nano hybrid system for biosensing applications," in Proceedings of the Mechanical Engineering and Engineering Mechanics, Michigan Technological University, Houghton, Mich, USA, 2008.

[19] P. S. K. Karre, "Fabrication and charaterization of room temperature operating single electron transistors using focused ion beam technologies," in Electrical Engineering, p. 188, Michigan Technological University, Houghton, Mich, USA, 2008.

[20] W. W. Wang, G. K. Knopf, and A. S. Bassi, "Photoelectric properties of a detector based on dried bacteriorhodopsin film," Biosensors and Bioelectronics, vol. 21, no. 7, pp. 1309-1319, 2006.

[21] J. Greer, A. Korkin, and J. Labanowski, Nano and Giga Challenges in Microelectronics, Elsevier, New York, NY, USA, 1st edition, 2003.

[22] R. Garcia, J. Tamayo, J. M. Soler, and C. Bustamante, "Physical parameters that control the imaging of purple membranes with the scanning tunneling microscope," Langmuir, vol. 11, no. 6, pp. 2109-2114, 1995.

[23] Y. Jin, N. Friedman, M. Sheves, T. He, and D. Cahen, "Bacteriorhodopsin (bR) as an electronic conduction medium: current transport through bR-containing monolayers," Proceedings of the National Academy of Sciences of the United States of America, vol. 103, no. 23, pp. 8601-8606, 2006.

[24] Y. Jin, T. Honig, I. Ron, N. Friedman, M. Sheves, and D. Cahen, "Bacteriorhodopsin as an electronic conduction medium for biomolecular electronics," Chemical Society Reviews, vol. 37, no. 11, pp. 2422-2432, 2008.

[25] P. S. K. Karre, M. Acharya, W. R. Knudsen, and P. L. Bergstrom, "Single electron transistor-based gas sensing with tungsten nanoparticles at room temperature," IEEE Sensors Journal, vol. 8, no. 6, pp. 797-802, 2008.

[26] D. D. Cheam, P. S. K. Karre, M. Palard, and P. L. Bergstrom, "Step and flash imprint lithography for quantum dots based room temperature single electron transistor fabrication," $M i$ croelectronic Engineering, vol. 86, no. 4-6, pp. 646-649, 2009.

[27] M. Acharya, Development of Room Temperature Operating Single Electron Transistors by FIB Etch and Deposition Process, Michigan Technological Univeristy, Houghton, Mich, USA, 2009. 

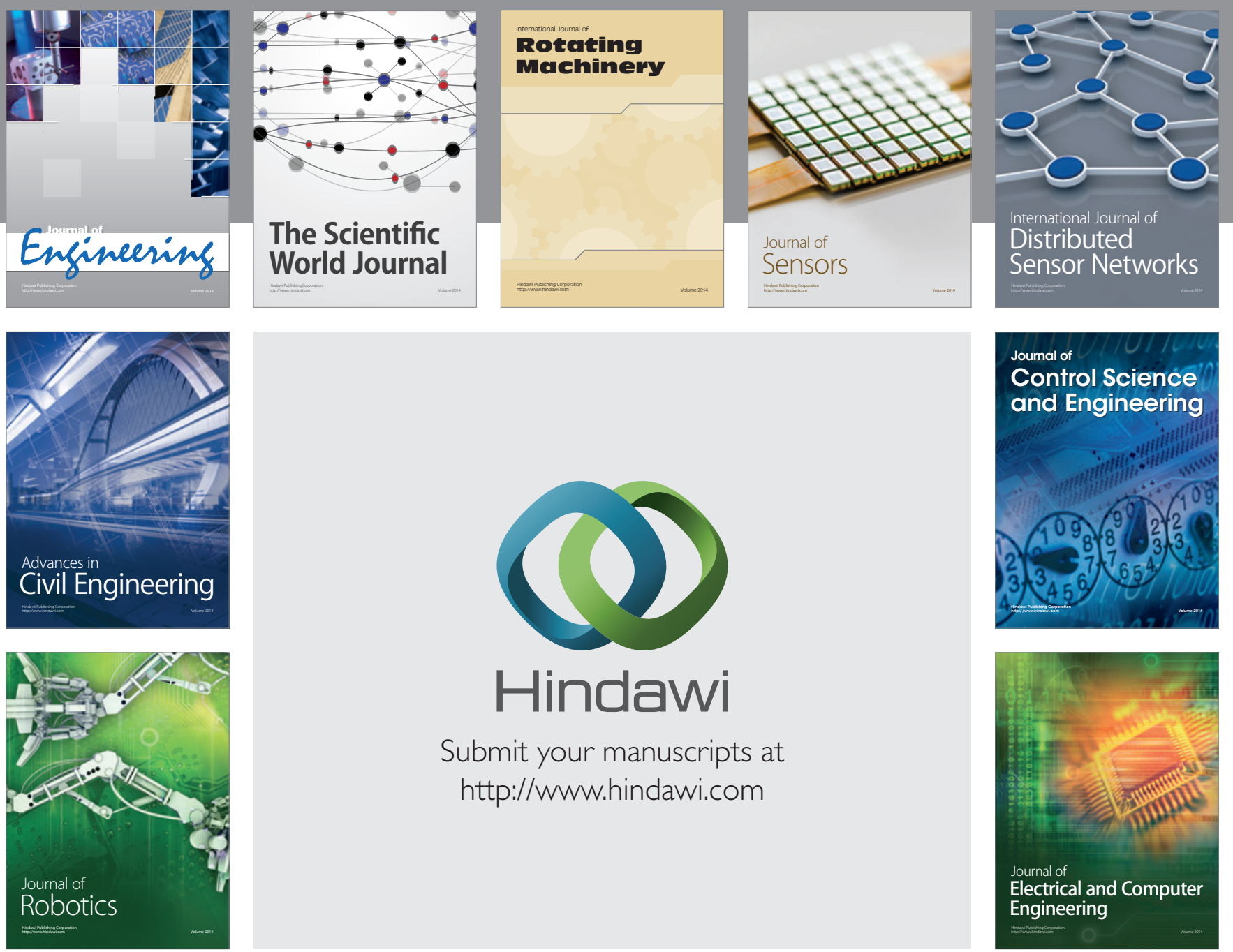

Submit your manuscripts at

http://www.hindawi.com
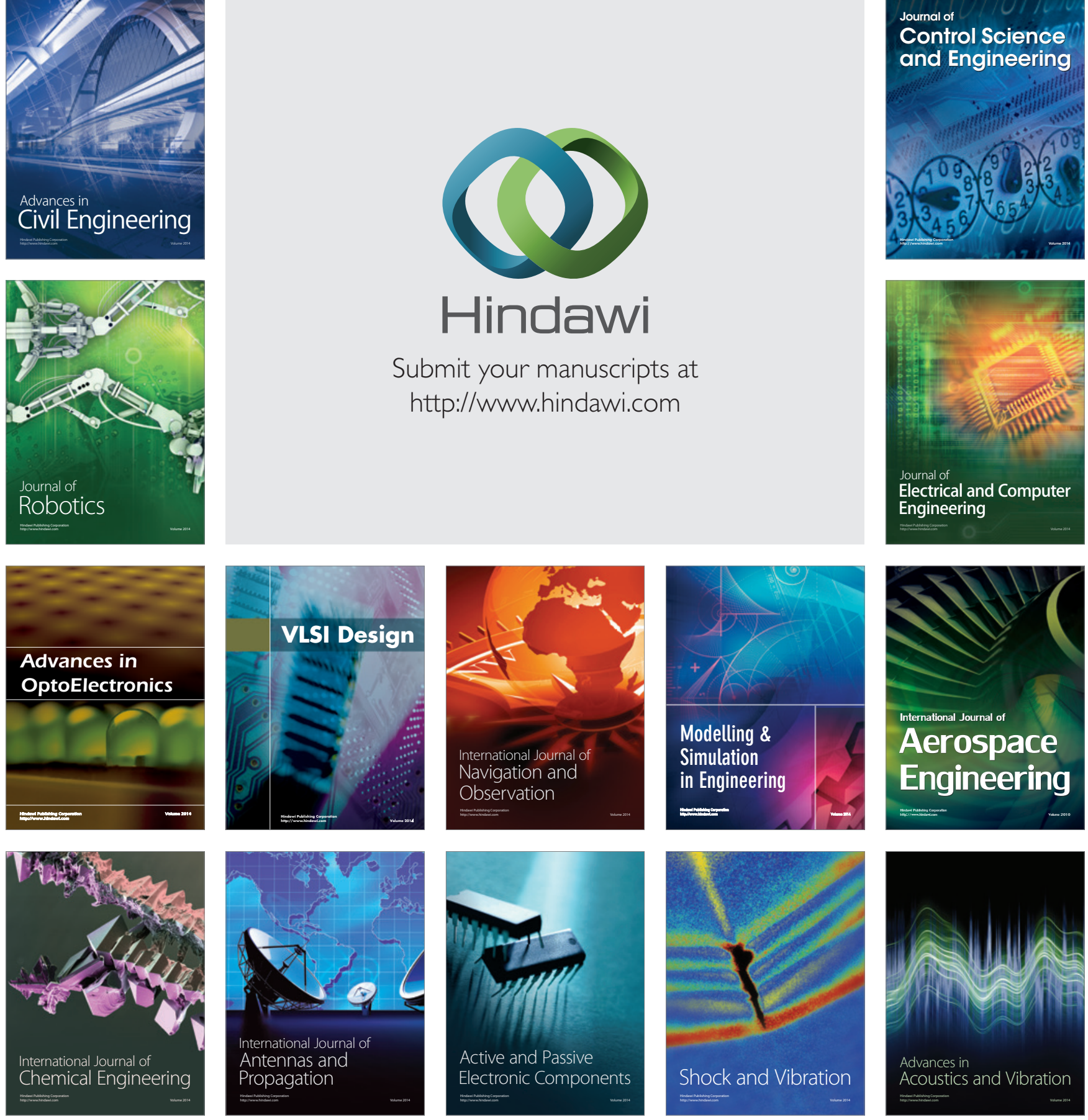